\title{
Entropy Stable Multi-dimensional Dissipation Function for the Roe Scheme on Unstructured Meshes
}

Conference Paper · January 2012

DOI: $10.2514 / 6.2012-569$

CITATIONS

0

2 authors:

\section{Aleksandar Jemcov \\ University of Notre Dame}

47 PUBLICATIONS 152 CITATIONS

SEE PROFILE
READS

46

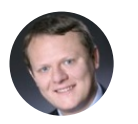

Hrvoje Jasak

University of Zagreb

121 PUBLICATIONS $\quad 2,460$ CITATIONS

SEE PROFILE

Some of the authors of this publication are also working on these related projects:

Project Numerical Modelling of Coupled Potential and Viscous Flow for Marine Applications View project

Project
Breaking the Code of Breaking Waves View project

All content following this page was uploaded by Aleksandar Jemcov on 23 April 2015. 


\title{
Entropy Stable Multi-dimensional Dissipation Function for the Roe Scheme on Unstructured Meshes
}

\author{
$\underline{\text { Aleksandar Jemcov}^{*} \quad \underline{\text { Hrvoje Jasak }}}{ }^{\dagger}$
}

Flux difference schemes based on the Roe approximate Riemann solver provide for sharp resolution of shock waves and contact discontinuities. Despite its good properties, the Roe scheme does not possess entropy stability that is required for the scheme to provide physically correct solutions. In addition, since the approximate Riemann solver was developed using one-dimensional considerations, the carbuncle instability appears around stagnation points for mesh-aligned flows. A solution to both entropy stability and the carbuncle problem is considered here. An alternative form of the dissipation function is developed that addresses both problems. The new dissipation function is based on the multidimensional modification to dissipation function of Roe scheme. The new scheme is shown to maintain sharp resolution comparable to the Roe flux difference scheme while satisfying entropy stability and removing the carbuncle type of instability.

\section{Nomenclature}

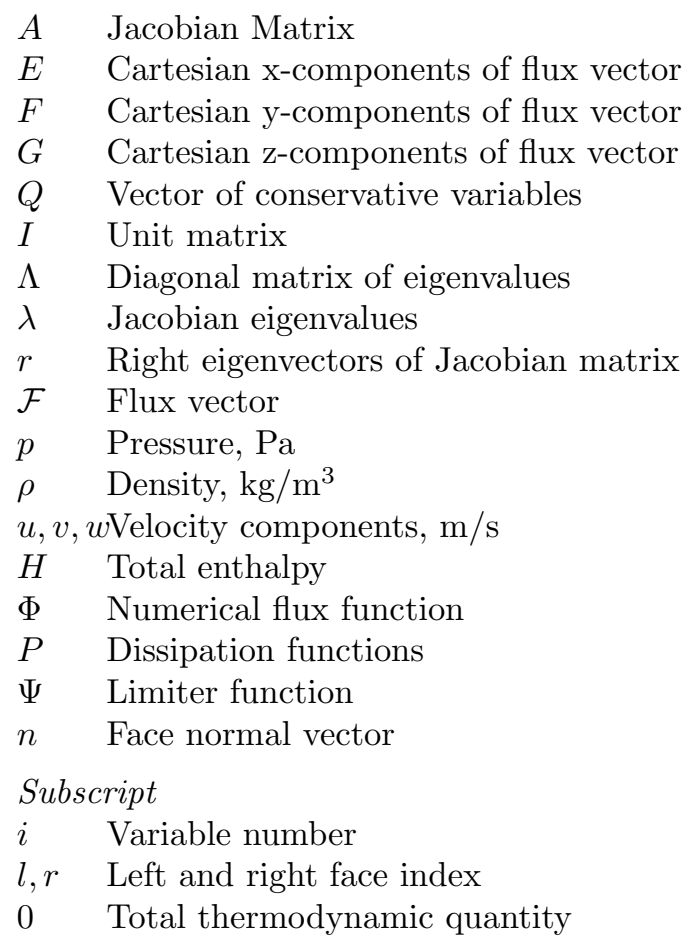

\section{Introduction}

The Roe flux difference splitting scheme ${ }^{1}$ is characterized by a sharp resolution of waves in non-linear and linear hyperbolic time dependent problems. The approximate Riemann solver of Roe employs linear

*Independent Consultant, 67 Melanson Lane, Brookside, Nova Scotia, B3T 2K9, Canada.

${ }^{\dagger}$ Director, Wikki Ltd., 31 Dolben Court Montaigne Close, London, SW1P 4BB, UK 
waves and the Roe averaging procedure to compute non-linear waves produced by non-linear flux functions. The resulting numerical flux is very well suited for the resolution of discontinuous solutions and it is often employed in computational fluid dynamics as the Riemann solver of choice. Also, the Roe scheme is known not to be entropy stable, and it can produce unphysical solutions in the vicinity of sonic points. A typical example of an unphysical solution that is characteristic of the Roe scheme is the appearance of expansion shocks in violation of entropy stability. Several approaches to remedy the situation have been proposed in the past ${ }^{2,3}$ through so-called entropy correction modifications. While these proposals are successful in removing the non-physical solutions, thus providing the entropy stability to the scheme, they still suffer from instabilities in the vicinity of stagnation points, often referred to as the carbuncle phenomenon. ${ }^{4}$ While carbuncle instability under certain circumstances represents a correct vanishing viscosity solution, ${ }^{5}$ the phenomenon is usually considered harmful, leading to an incorrect physical solution. The carbuncle type of instability appears in the solution of Euler equations in more than one space dimension. The instability is linked to the lack of cross flow dissipation that is typical of high resolution upwind one-dimensional schemes such as Roe scheme, when applied to multi-dimensional flow problems.

At the same time, it is well known that the numerical schemes based on a central differencing approach do not suffer from the carbuncle or entropy instabilities. ${ }^{3}$ The classical examples of schemes that are based on central differencing formulas are Lax-Friedrichs, local Lax-Friedrichs, and JST schemes. ${ }^{3,6}$ All of these schemes are characterized by increased dissipation that is of multi-dimensional nature. While JST scheme satisfies the entropy stability condition ${ }^{7}$ we limit the analysis to Lax-Friedrichs and local Lax-Friedrichs schemes since they provide a form of the numerical fluxes that can be easily related to the Roe scheme.

The main idea employed here is to modify the dissipation function of the Roe flux difference formula so that it has some of the favorable dissipation characteristics of Lax-Friedrichs and local Lax-Friedrichs schemes, while maintaining a sharp resolution of discontinuities. The resulting numerical flux is still of the Roe type with the modified dissipation function, so that the entropy and multi-dimensional stability requirements are satisfied.

\section{Governing Equations and Numerical Method}

Inviscid compressible fluid flow is described by Euler equations

$$
\frac{\partial \mathbf{Q}}{\partial t}+\frac{\partial \mathbf{E}}{\partial x}+\frac{\partial \mathbf{F}}{\partial y}+\frac{\partial \mathbf{G}}{\partial z}=0
$$

where $\mathbf{Q}$ is the vector of conserved variables

$$
\mathbf{Q}=\left(\begin{array}{c}
\rho \\
\rho u \\
\rho v \\
\rho w \\
\rho E
\end{array}\right)
$$

and $\mathbf{E}$ and $\mathbf{F}$ are vectors of inviscid fluxes

$$
\mathbf{E}=\left(\begin{array}{c}
\rho u \\
\rho u^{2}+p n_{x} \\
\rho u v \\
\rho u w \\
\rho u H
\end{array}\right), \quad \mathbf{F}=\left(\begin{array}{c}
\rho v \\
\rho u v \\
\rho v^{2}+p n_{y} \\
\rho v w \\
\rho v H
\end{array}\right), \quad \mathbf{G}=\left(\begin{array}{c}
\rho w \\
\rho u w \\
\rho v w \\
\rho w^{2}+p n_{z} \\
\rho v H
\end{array}\right)
$$

and $u, v, w, p, E, H$ have their usual meaning of Cartesian components of velocity, static pressure, total energy and total enthalpy, respectively. Operator $\mathcal{B}$ acting on vector variable $\mathbf{Q}$ defines the inviscid boundary conditions. In the particular example of interest here, wall boundary conditions are described by the nopenetration condition

$$
u n_{x}+v n_{y}+w n_{z}=0
$$


and inlet and outlet boundary conditions are prescribed in accordance with the sign of characteristics with the following relationships between total and static quantities

$$
\begin{gathered}
\frac{T_{o}}{T}=1+\frac{\gamma-1}{2} M^{2} \\
\frac{p_{o}}{p}=\left(1+\frac{\gamma-1}{2} M^{2}\right)^{\gamma /(\gamma-1)} \\
\frac{\rho_{o}}{\rho}=\left(1+\frac{\gamma-1}{2} M^{2}\right)^{1 /(\gamma-1)}
\end{gathered}
$$

Here $n_{x} n_{y}$, and $n_{z}$ are Cartesian components of the unit normal vector of the boundary $\partial \Omega, M$ is the Mach number and all quantities with subscript " $o$ " represent total thermodynamic quantities. Eq. (1) can be written in compact form using index notation

$$
\frac{\partial \mathbf{Q}}{\partial t}+\frac{\partial \mathcal{F}_{i}(\mathbf{Q})}{\partial x_{i}}=0 .
$$

Here $\mathcal{F}_{i}$ are components of the flux vector and $x_{i}$ are components of a vector of Cartesian coordinates.

In finite volume methods Eq. (5) is transformed into a weak form through integration

$$
\int_{\Omega} \frac{\partial \mathbf{Q}}{\partial t}+\int_{\Omega} \frac{\partial \mathcal{F}_{i}(\mathbf{Q})}{\partial x_{i}}=0 .
$$

Application of Green's theorem to Eq. (6) results in the following weak form containing the surface terms

$$
\int_{\Omega} \frac{\partial \mathbf{Q}}{\partial t}+\int_{\Gamma} \mathcal{F}_{i}(\mathbf{Q}) n_{i}=0 .
$$

If the whole computational domain is discretized in non-overlapping control volumes $\Omega_{i}$, the weak form in Eq. (7) for each finite volume takes the following form:

$$
\int_{\Omega_{i}} \frac{\partial \mathbf{Q}}{\partial t}+\sum_{i} \int_{\Gamma_{i}} \mathcal{F}_{i}(\mathbf{Q}) n_{i}=0
$$

where the sum is taken over all faces of finite volume $\Omega_{i}$.

The numerical flux function $\Phi\left(\mathbf{Q}_{l}, \mathbf{Q}_{r}\right)$ is introduced into Eq. (8) in order to evaluate surface integrals

$$
\sum_{i} \int_{\Gamma_{i}} \mathcal{F}_{i}(\mathbf{Q}) n_{i} \approx \sum_{i} \Phi_{i}\left(\mathbf{Q}_{l_{i}}, \mathbf{Q}_{r_{i}}, n_{i}\right) .
$$

Subscripts $l$ and $r$ correspond to "left" and "right" states relative to the face $\Gamma_{i}$ and the numerical flux function $\Phi_{i}$ defined through a local Riemann problem evaluated at integration points on the face. The general form of numerical flux function $\Phi_{i}$, suitable for description of both upwind and central difference schemes, is given by

$$
\Phi_{i}\left(\mathbf{Q}_{l}, \mathbf{Q}_{r}, n\right)=\frac{1}{2}\left(\mathcal{F}_{l}+\mathcal{F}_{r}\right) n-\frac{1}{2} P\left(\mathbf{Q}_{l}, \mathbf{Q}_{r}, n\right) .
$$

Various schemes take a different form of the dissipation function $P\left(\mathbf{Q}_{l}, \mathbf{Q}_{r}, n\right)$ depending on whether they are based on upwind flux difference splitting or the central scheme. In the case of the Roe scheme, the dissipation function takes the following form:

$$
P\left(\mathbf{Q}_{l}, \mathbf{Q}_{r}, n\right)=\left|A\left(\overline{\mathbf{Q}}_{f}, n_{f}\right)\right|\left(\mathbf{Q}_{r}-\mathbf{Q}_{l}\right),
$$

or equivalently after spectral decomposition of Jacobian $A$ :

$$
P\left(\mathbf{Q}_{l}, \mathbf{Q}_{r}, n\right)=\left|\Lambda\left(\overline{\mathbf{Q}}_{f}\right)\right| \mathbf{r}\left(\mathbf{Q}_{r}-\mathbf{Q}_{l}\right) .
$$

Lax-Friedrichs numerical flux can be cast in the form given by Eq. (10) by noting that the dissipation function of that scheme is given by the following expression

$$
P\left(\mathbf{Q}_{l}, \mathbf{Q}_{r}, n\right)=\lambda_{\max }\left(\mathbf{Q}_{r}-\mathbf{Q}_{l}\right),
$$


where

$$
\lambda_{\max }=\max _{\lambda, \mathbf{Q}}(\lambda(\mathbf{Q})) .
$$

The resulting Lax-Friedrichs scheme has a dissipation function with uniform viscosity coefficient $\lambda_{\max }$. This numeric flux function is known to be very dissipative. Similarly, the local Lax-Friedrics flux dissipation function is given by

$$
P\left(\mathbf{Q}_{l}, \mathbf{Q}_{r}, n\right)=\lambda_{l r \max }\left(\mathbf{Q}_{r}-\mathbf{Q}_{l}\right),
$$

where

$$
\lambda_{l r \max }=\max _{\lambda_{l, r}, \mathbf{Q}_{l}, \mathbf{Q}_{r}}(\lambda(\mathbf{Q})) .
$$

Instead of selecting one uniform dissipative coefficient as in Eq. (14), the local Lax-Friedrichs scheme selects a non-uniform cell based dissipation coefficient in Eq. (16), thus providing for a less dissipative numerical flux function. It is also known that both Lax-Fiedrichs and local Lax-Friedrichs schemes are entropy stable with respect to any entropy pair associated with Eq. (5). Moreover, due to the multi-dimensional nature of the dissipation function, both schemes are free of the carbuncle phenomenon.

In contrast to dissipation functions in Eq. (14) and (16), the Roe dissipation function does not satisfy the discrete entropy stability condition

$$
\frac{d}{d t} \mathbf{U}(\mathbf{Q})+\frac{1}{V} \sum_{i} \mathcal{G}_{i} n_{i} \leq 0
$$

for arbitrary entropy pair $(U, \mathcal{G})$. This situation can be remedied by introducing the following entropy stable dissipation function ${ }^{3}$

$$
P\left(\mathbf{Q}_{l}, \mathbf{Q}_{r}, n\right)=\max \left\{\left|\lambda_{l r}\right|, K C_{l r}^{1}\left(\mathbf{Q}_{r}-\mathbf{Q}_{r}\right)\right\},
$$

or

$$
P\left(\mathbf{Q}_{l}, \mathbf{Q}_{r}, n\right)=\left|\lambda_{l r}\right|+\left[\frac{1}{6}\left(\lambda_{r}-\lambda_{l}\right)+C_{l r}^{2}\left(\mathbf{Q}_{r}-\mathbf{Q}_{r}\right)^{2}\right] .
$$

The modifications in Eq. (18) and (19) are sufficient to provide entropy stability and avoid unphysical solutions for sufficiently large choice of constants $K, C_{l r}^{1}$, and/or $C_{l r}^{2}$. However, expressions in Eq. (18) and (19) are derived with one-dimensional numeric flux functions in mind, and they do not address the lack of cross diffusion in the dissipation function that leads to the carbuncle instability.

One possible way of addressing both entropy stability and carbuncle problems in the Roe scheme is to use the general form of the numerical flux function Eq. (10) to take advantage of the local Lax-Friedrichs formulation of the dissipation function, and bring its stability to the Roe dissipation function. That can be done if the new dissipation function takes the following form:

$$
P\left(\mathbf{Q}_{l}, \mathbf{Q}_{r}, n\right)=\left[\alpha_{1}\left|\Lambda\left(\overline{\mathbf{Q}}_{f}\right)\right| \mathbf{r}+\alpha_{2} \max _{\lambda_{l, r}, \mathbf{Q}_{l}, \mathbf{Q}_{r}}(\lambda(\mathbf{Q})) \mathbf{I}\right]\left(\mathbf{Q}_{r}-\mathbf{Q}_{l}\right) .
$$

Two arbitrary parameters $\alpha_{1}$ and $\alpha_{2}$ appear in Eq. (20. They have to be selected so that the entropy stability requirement in Eq. (17) is satisfied. However, it is also important that the selection of the constants does not bring too much viscosity into the dissipation function since this would destroy the good properties of the Roe scheme. Therefore, it is required that the numerical dissipation in Eq. (20) does not exceed the numerical dissipation of the local Lax-Friedrichs scheme in Eq. (16). This requirement clearly indicates that parameters $\alpha_{1}$ and $\alpha_{2}$ should not be chosen independently. In order to satisfy the stated requirement, one possible choice of the dissipation function could be the following expression:

$$
P\left(\mathbf{Q}_{l}, \mathbf{Q}_{r}, n\right)=\left[\left|\Lambda\left(\overline{\mathbf{Q}}_{f}\right)\right| \mathbf{r}+K\left|\max _{\lambda_{l, r}, \mathbf{Q}_{l}, \mathbf{Q}_{r}}(\lambda(\mathbf{Q})) \mathbf{I}-\right| \Lambda\left(\overline{\mathbf{Q}}_{f}\right)|\mathbf{r}|\right]\left(\mathbf{Q}_{r}-\mathbf{Q}_{l}\right), \quad K \in\{0,1\} .
$$

Constraining the constant $K$ to the interval $\{0,1\}$ assures that the dissipation function never exceeds the value of the local Lax-Friedrichs dissipation. The value of the the constant $K$ is numerically determined and may vary from problem to a problem. However, once selected, it should be valid for a wide range of Mach numbers. 


\section{Numerical Experiments}

In order to demonstrate the newly defined dissipation function, numerical experiments were performed for flows known to contain expansion waves and stagnation points. One such problem is described in Woodward and Collela. ${ }^{8}$ It consists of supersonic flow in a wind tunnel with a step, as shown in figure 1 . This is a two-dimensional problem that has uniform Mach 3 flow at the inlet of the wind tunnel. The wind tunnel is 1 length unit wide and 3 length units long, with a step. The flow field is characterized by the presence of a strong bow shock close to the step, expansion wave around the corner, reflected waves and slip surfaces. Since there is a strong stationary bow shock and expansion wave in the flow field of this problem, both entropy stability and the carbuncle phenomenon are tested by this case.
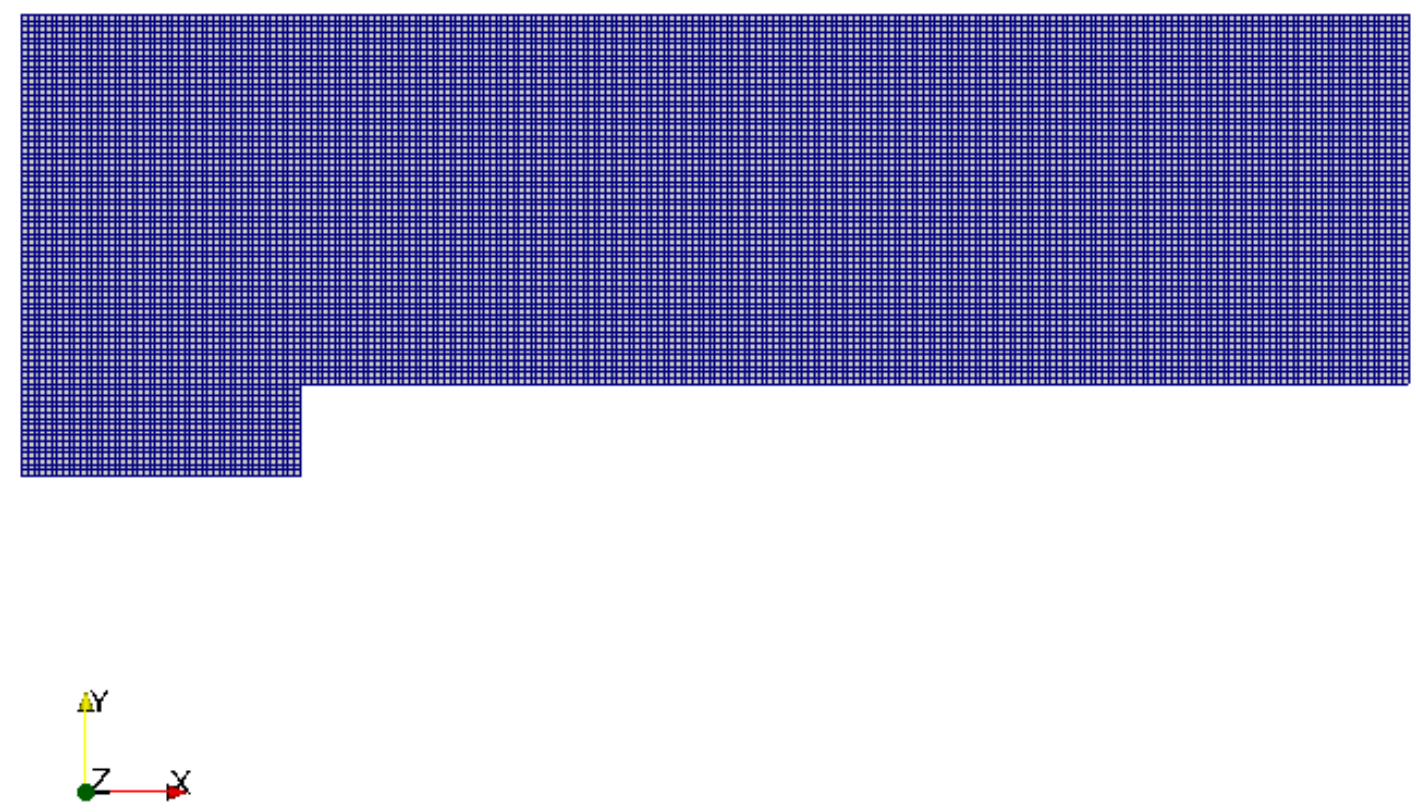

Figure 1. Computational mesh for the Mach 3 wind tunnel with a step. The mesh size correspond to spacing $\triangle x=$ $\triangle y=1 / 80$.

An explicit time marching algorithm using a four stage Runge-Kutta scheme was used to compute the flow field at various times. A cell centered finite volume discretization was used to obtain the spatial discretization. In order to obtain second order accuracy, primitive variables were extrapolated to the centroids of the finite volume faces in accordance with the following expression

$$
\phi_{f}=\phi_{c}+\Psi \vec{r} \cdot \nabla \phi .
$$

A Multidimensional limiter function $\Psi$ is employed to guarantee stability of the second order scheme. ${ }^{9}$ 
Pressure and density distributions are shown in figure (2) and figure (3 at the time $t=3$.
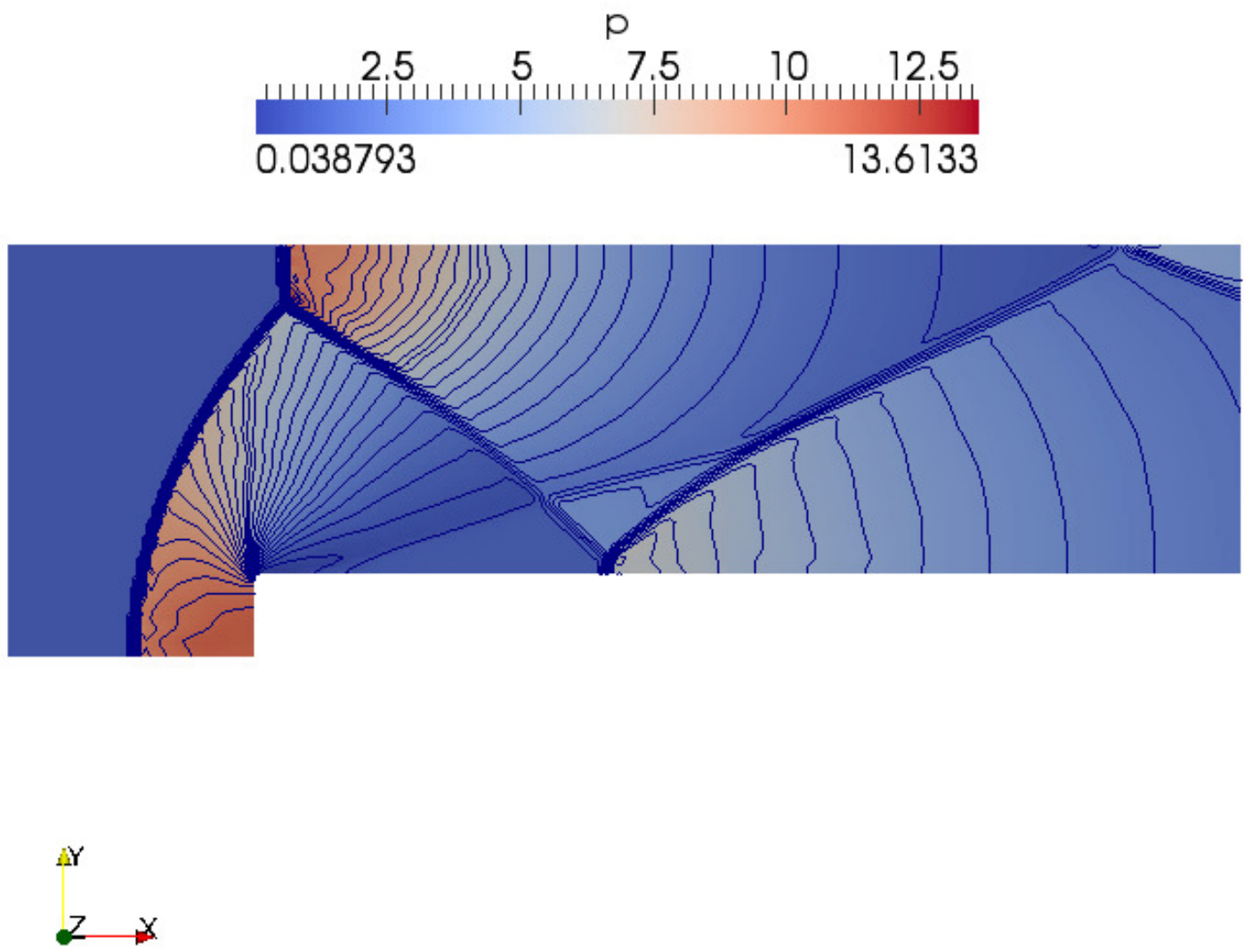

Figure 2. Pressure distribution at time $T=3$ computed by the Roe scheme. The mesh size correspond to spacing $\triangle x=\triangle y=1 / 80$.

Close inspection of figure (2) and figure (3 reveals significant fluctuations in both pressure and density just behind the stationary leading shock wave and stagnation point. The ripples in fields are a sign of the instability caused by the carbuncle phenomenon. This effect can be seen closely in figure (4) in the case of the density field computed on higher density grid $(\triangle x=\triangle y=1 / 160)$.

In contrast to Roe flux differencing, the local Lax-Friedrichs scheme shows no signs of carbuncle or entropy instability. Pressure and density fields are shown in figure (6) and figure (5). However, due to increased viscosity, local Lax-Friedrichs does not resolve shock waves and other discontinuities in the flow field.

Even though the local Lax-Friedrichs scheme is second-order accurate and satisfies the entropy condition, it does not resolve discontinuities as sharply as the Roe scheme. However, it has superior stability characteristics in comparison to the Roe scheme. Details of the density field computed by the local Lax-Friedrichs scheme are shown in figure (7). The figure shows very smooth pressure and density fields, with satisfactory resolution of shock waves even though the results are somewhat dissipative.

Therefore, it is natural to try to combine the best characteristics of both schemes through Eq. (21). Numerical results show that the selection of parameter $K=0.01$ retains the usual entropy fix of Roe scheme, while increasing its robustness. However, if the entropy fix described $\mathrm{in}^{2}$ is replaced by the dissipation in Eq. 21) with the parameter $K=0.4$, essentially all of the sharp resolution of Roe scheme and stability of 

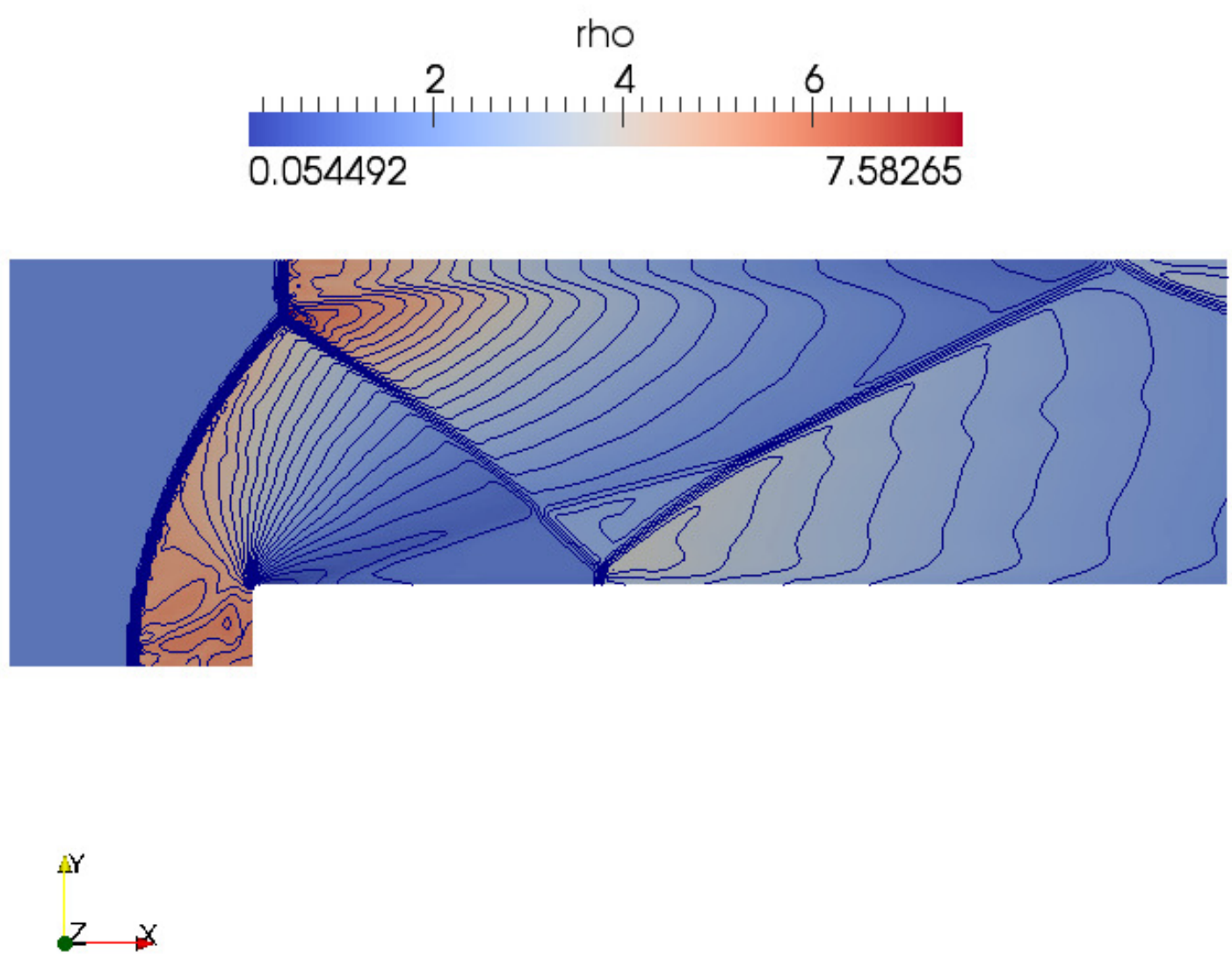

Figure 3. Density distribution at time $T=3$ computed by Roe scheme. The mesh size correspond to spacing $\triangle x=$ $\triangle y=1 / 80$.

the local Lax-Friedrichs are retained. Computational results shown in figure (9) and figure (8).

The details of the computed density field are shown in figure (10). The computed flow field is smooth behind the shock wave while the resolution of discontinuities is close to the Roe scheme. The modified Roe scheme satisfies both entropy and carbuncle stability conditions.

\section{Conclusion}

In the final version of the paper, more computational experiments will be described. Also additional analysis of the modified Roe scheme will be provided. All sections will be expanded significantly to provide more information on the details of the new method.

\section{References}

${ }^{1}$ Roe, P. L., Approximate Riemann Solver, Parameter Vectors, and Difference Schemes, J. Comp. Phys, 43 (1981), pp $357-372$.

${ }^{2}$ Harten, A. and Hyman, J., Self-adjusting grid method for one dimensional hyberbolic conservation laws, J. Comp. Phys, 50 (1983), pp 235-269. 


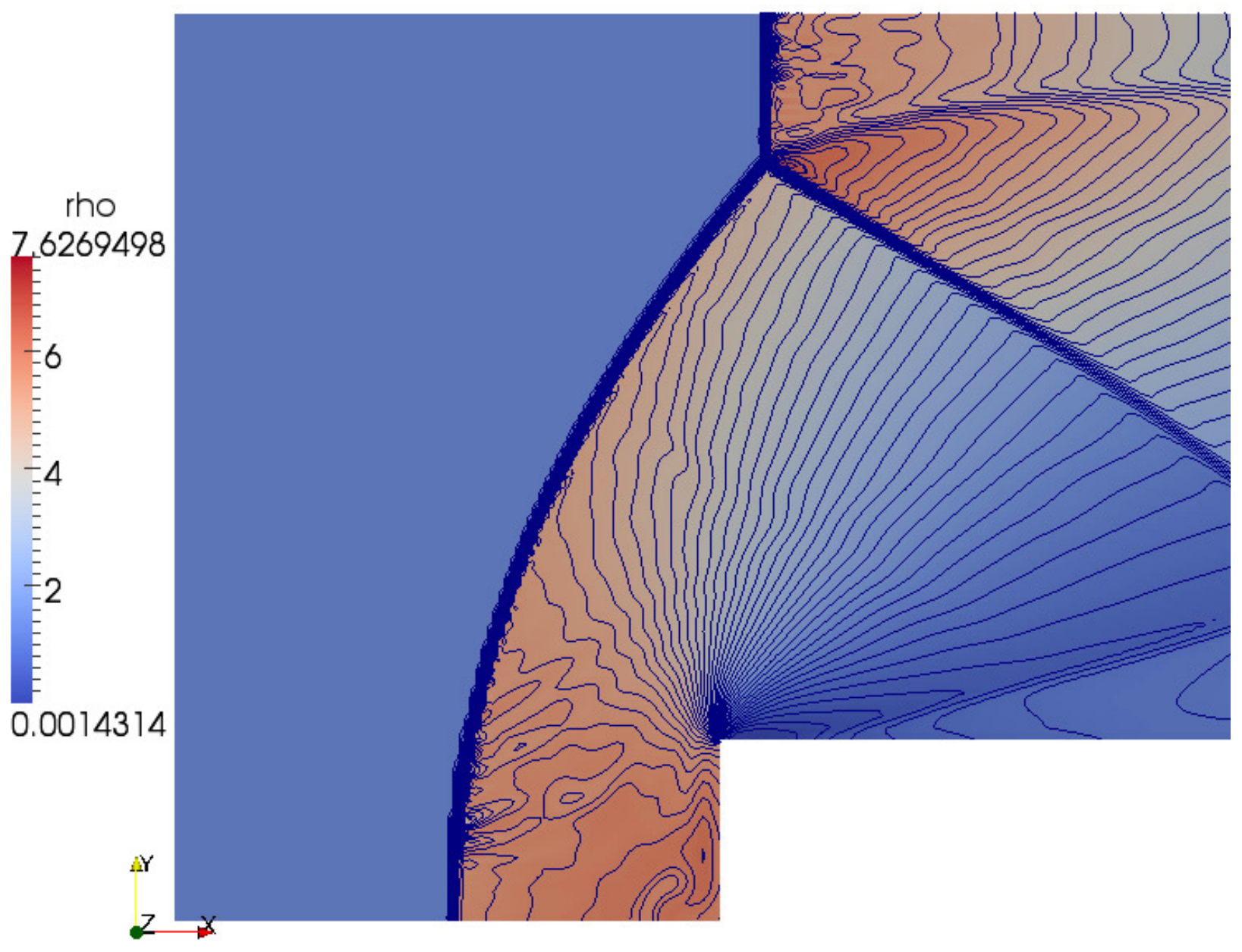

Figure 4. Density distribution at time $T=3$. Carbuncle instability is shown around leading shock wave and the stagnation point. The mesh size correspond to spacing $\triangle x=\Delta y=1 / 160$.

${ }^{3}$ Tadmor, E., Entropy stability theory for difference approximations of nonlinear conservation laws and related timedependent problem, Acta Numerica, (2003), pp 451-512.

${ }^{4}$ Perry, K. M. and Imlay, S. T., Blunt-body flow simulations, AIAA paper, 88-2904 (1988).

${ }^{5}$ Eling, V., The Carbuncle Phenomenon is Incurable, Acta Mathematica Scientia, 29(B)(6)(2009), pp 1647-1658.

${ }^{6}$ Jameson, A., Schmidt, W., and Turkel, E., Numerical Solutions of the Euler Equations by Finite Volume Methods with Runge-Kutta Time Stepping Schemes, AIAA paper, 81-1259 (1981).

${ }^{7}$ Jameson, A., Analysis and Design of Numerical Schemes for Gas Dynamics 1 Artificial Diffusion, Upwind Biasing, Limiters and Their Effect on Accuracy and Multigrid Convergence, NTERNATIONAL JOURNAL OF COMPUTATIONAL FLUID DYNAMICS, (4), pp 171-218 (1995).

${ }^{8}$ Woodward, P., and Collela, P. The Numerical Simulation of Two-Dimensional Fluid Flow with Strong Shocks, J. Comp. Phys, 54 (1984), pp 115-173.

${ }^{9}$ Venkatakrishnan, V., Convergence to steady state solutions of the Euler equations on unstructured grids with limiters. , J. Comp. Phys, 118(1) (1995), pp 120-130. 


\section{$\mathrm{p}$

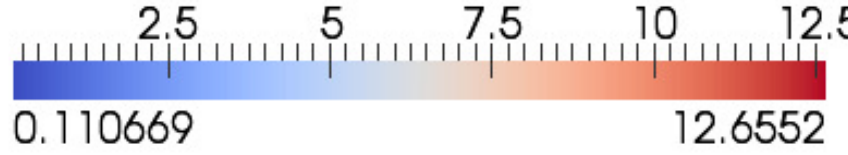
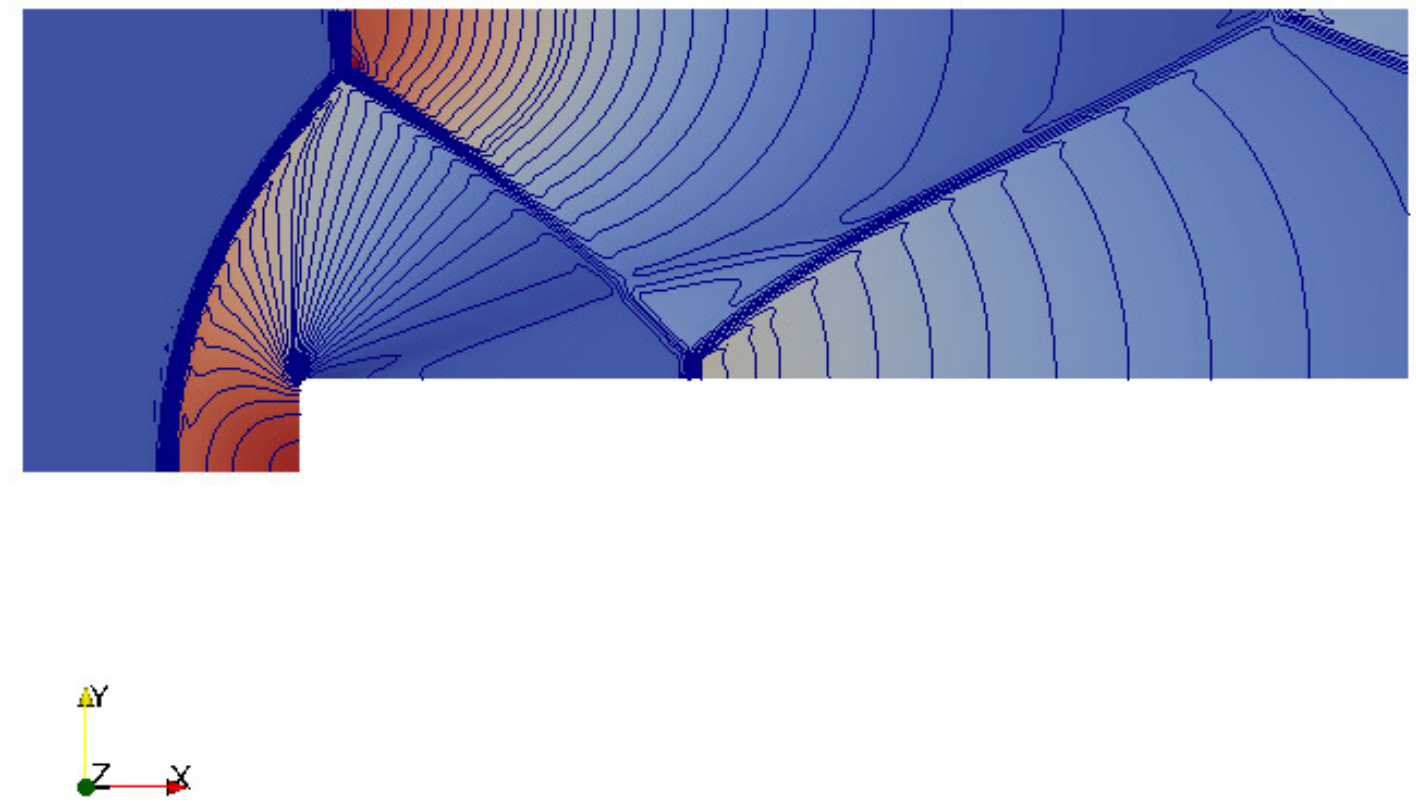

Figure 5. Pressure distribution at time $T=3$ computed by local Lax-Friedrichs scheme. The mesh size correspond to spacing $\triangle x=\triangle y=1 / 80$. 

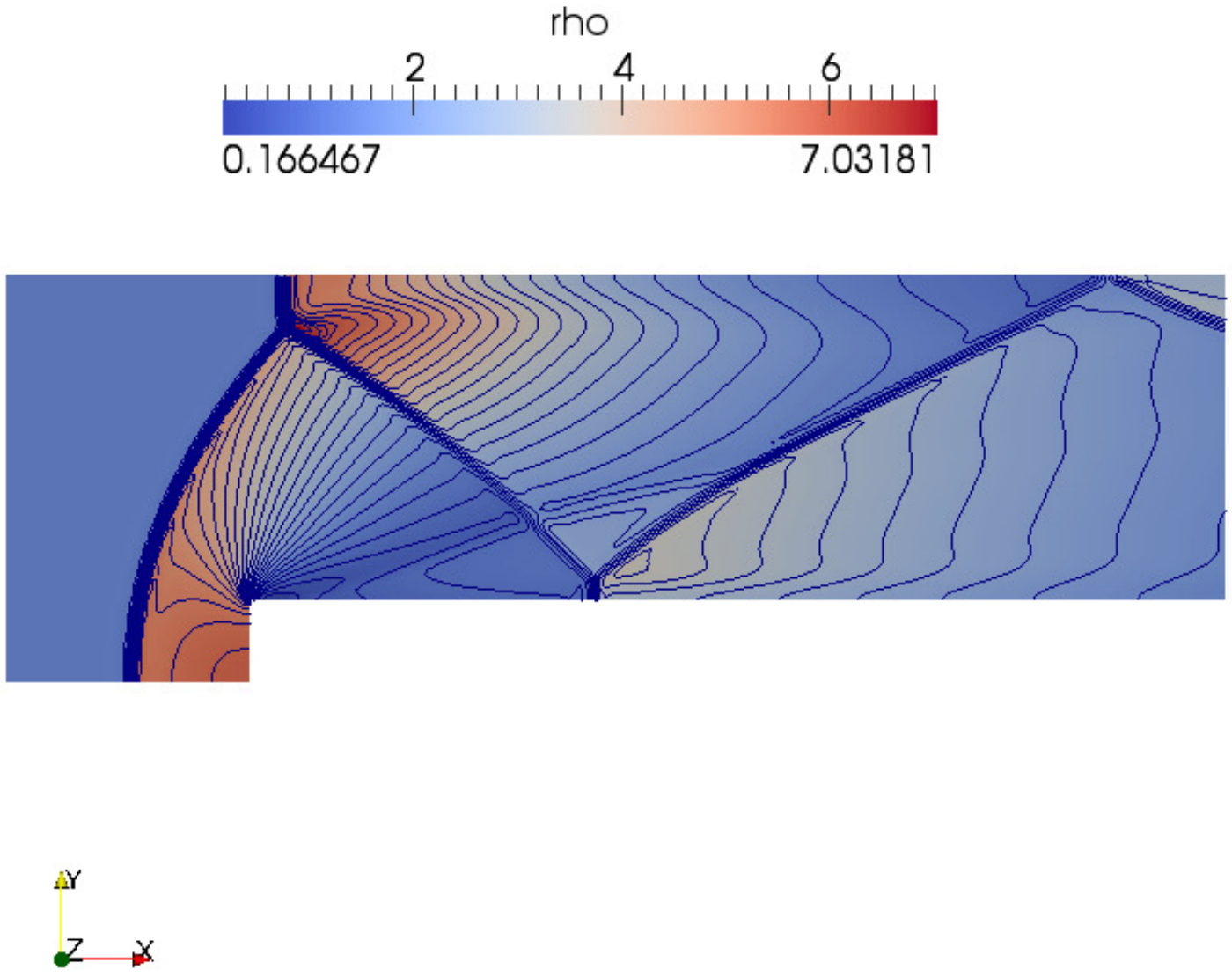

Figure 6. Density distribution at time $T=3$ computed by local Lax-Friedrichs scheme. The mesh size correspond to spacing $\triangle x=\triangle y=1 / 80$. 


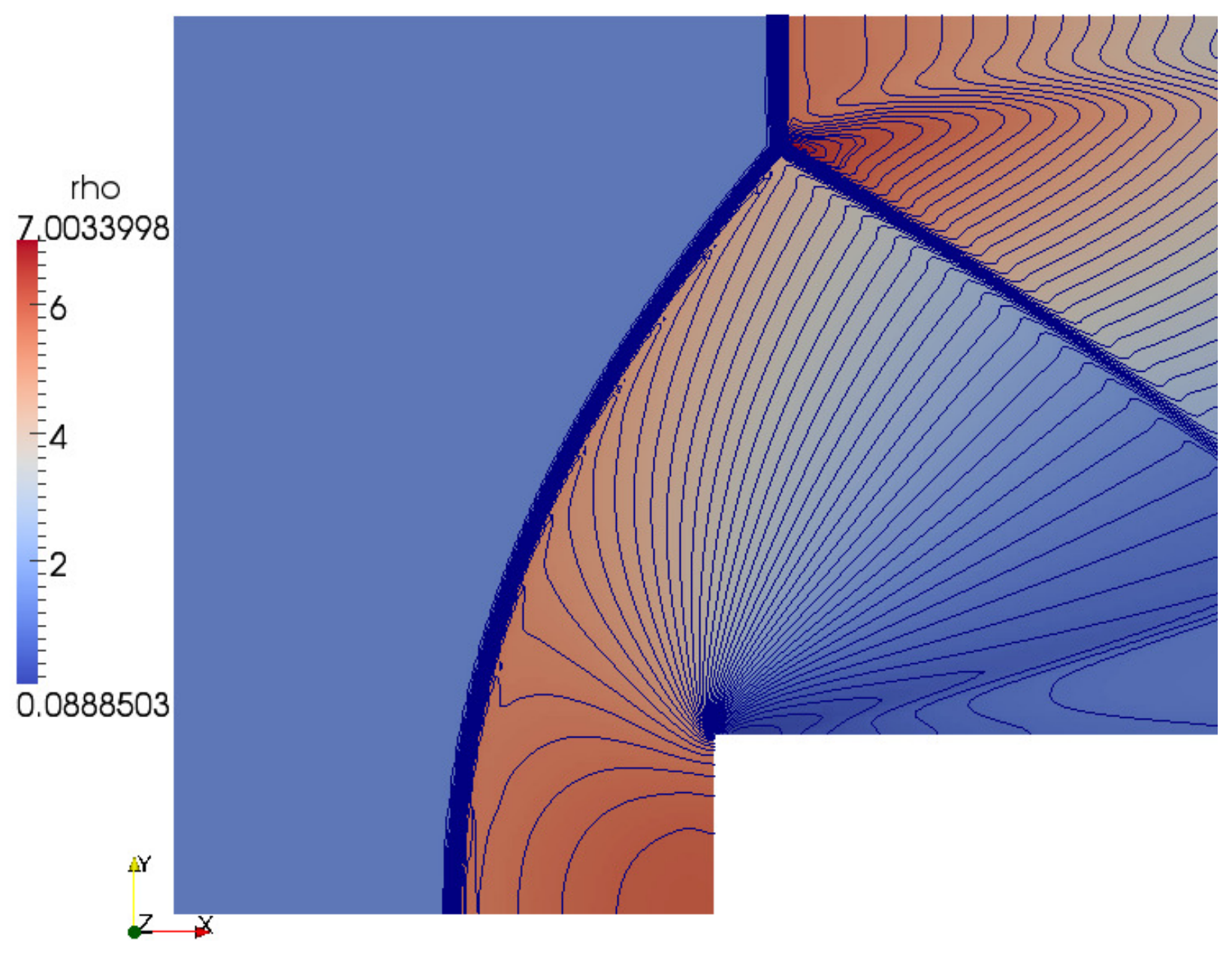

Figure 7. Density distribution at time $T=3$ computed by local Lax-Friedrichs scheme. The mesh size correspond to spacing $\triangle x=\triangle y=1 / 160$. 


\section{$2.5 \quad 5 \quad 7.5 \quad 10 \quad 12.5$

$0.019943 \quad 13.3652$
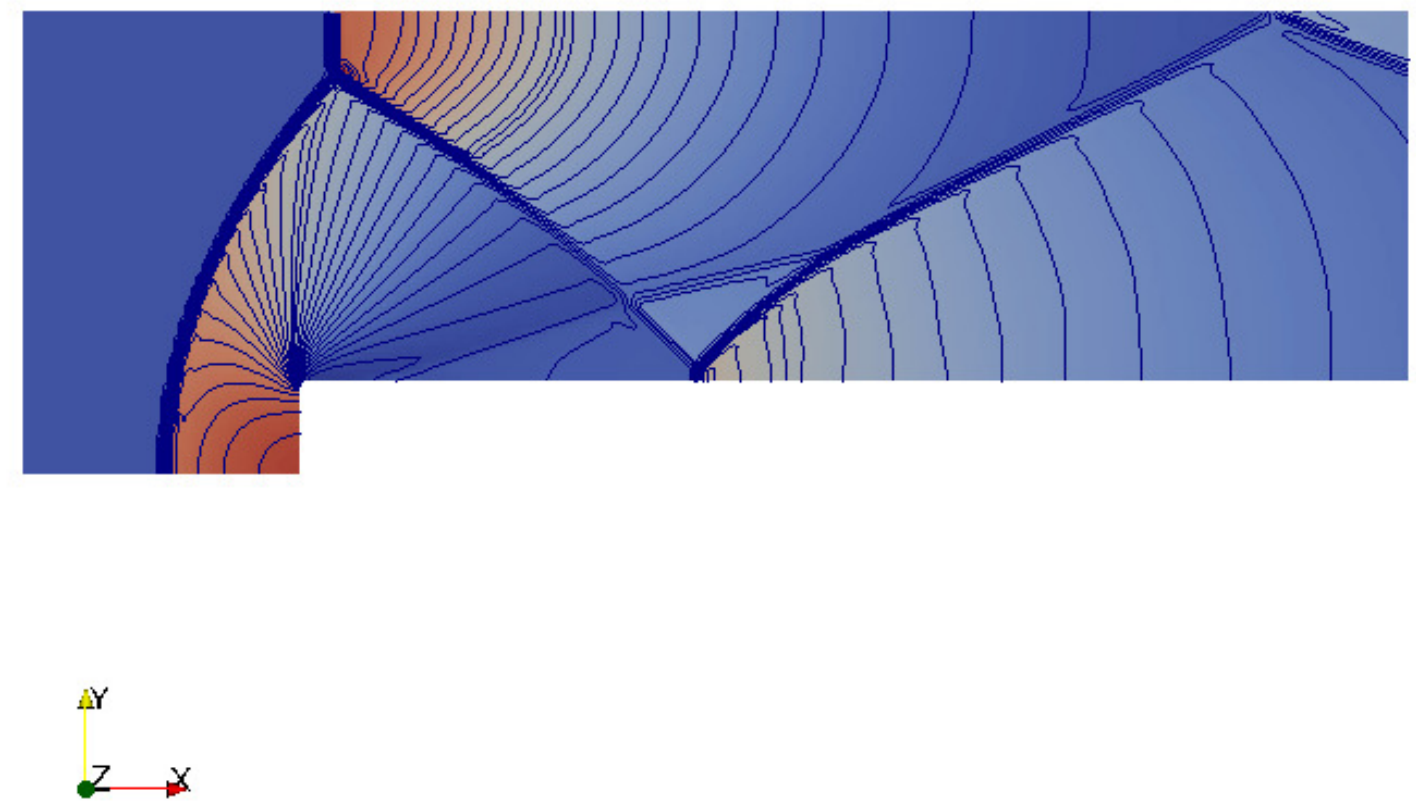

Figure 8. Pressure distribution at time $T=3$ computed by modified Roe scheme. The mesh size correspond to spacing $\triangle x=\triangle y=1 / 80$. 

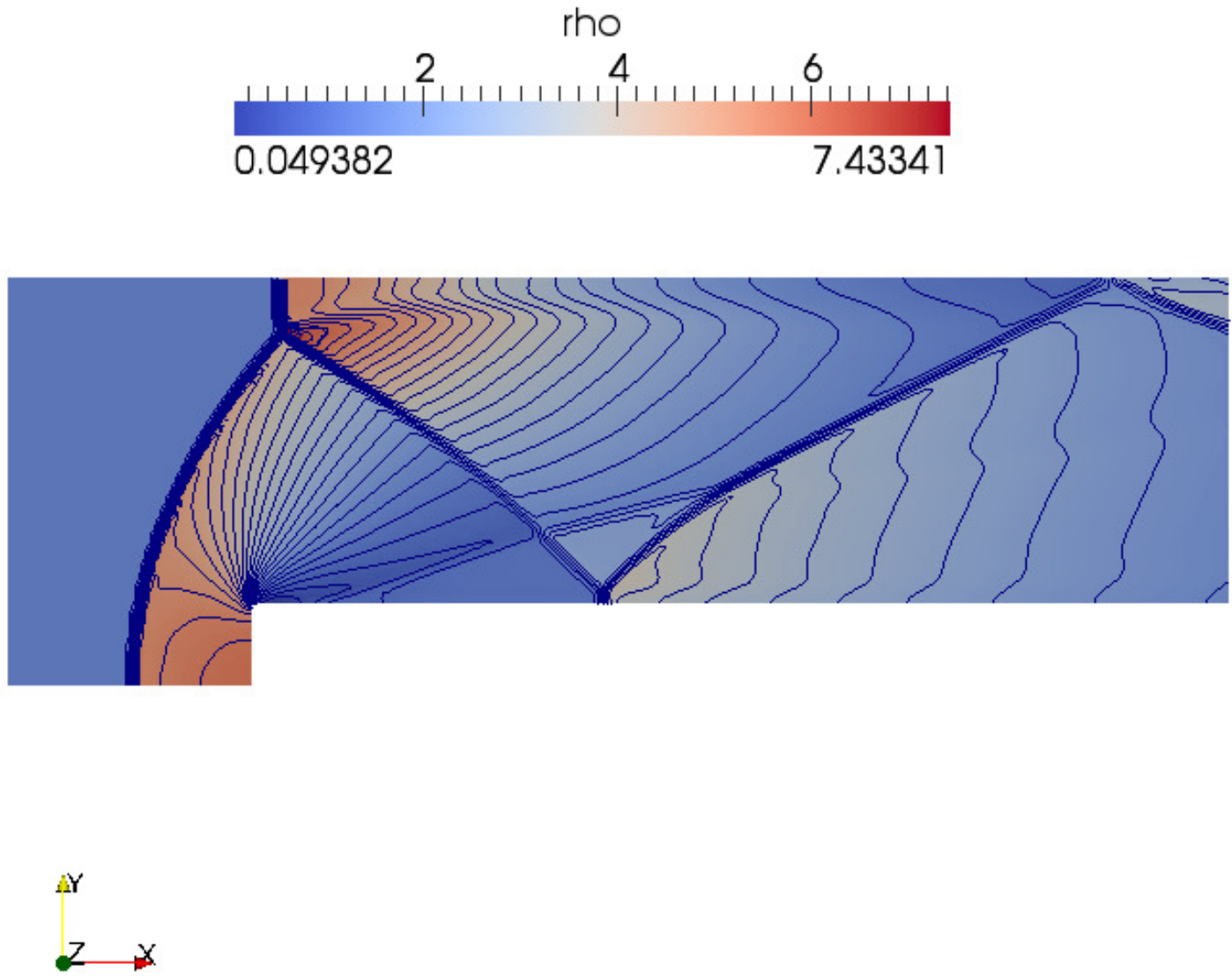

Figure 9. Density distribution at time $T=3$ computed by modified Roe scheme. The mesh size correspond to spacing $\triangle x=\triangle y=1 / 80$. 


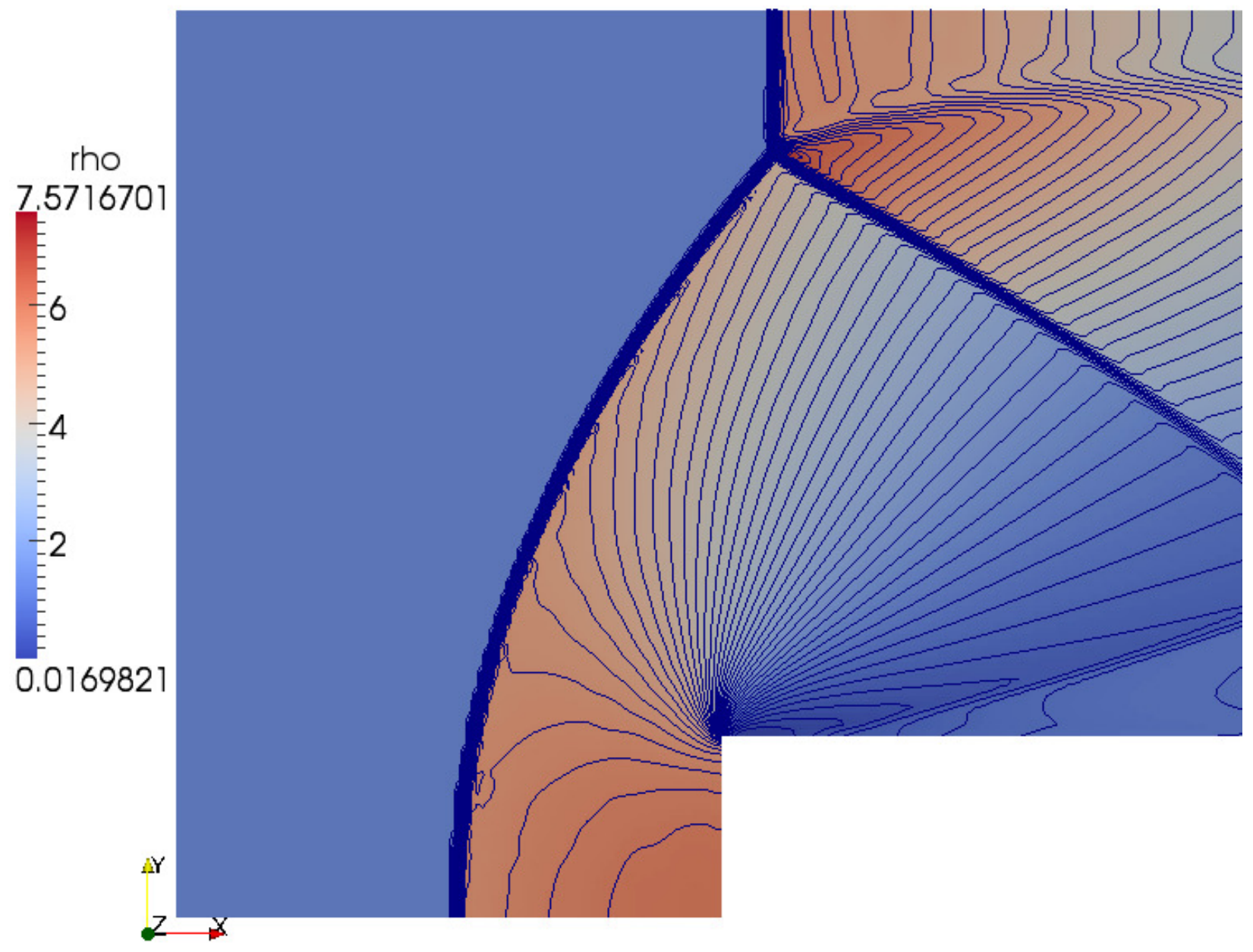

Figure 10. Density distribution at time $T=3$ computed by modified Roe scheme. The mesh size correspond to spacing $\triangle x=\triangle y=1 / 160$. 